Research Article

\title{
Development and Characterization of Eucalyptus Oil Loaded Poly Lactic Co-Glycolic Acid - Albumin Nanocapsules
}

\author{
C.Sadak Vali", Abdullah Khan ${ }^{1}$, M. Prathibha Bharathi ${ }^{2}$, S. Siva Prasad ${ }^{3}$, A. Srikanth ${ }^{4}$. \\ * Associate professor, Mohammadiya Institute of Pharmacy, Khammam, Telangana. India. \\ 1. KPJ Healthcare University College, Nilai, Malaysia. \\ 2. MNR College of Pharmacy, Hyderabad. Telangana. India. \\ 3. MNR College of Pharmacy, Hyderabad. Telangana. India. \\ 4. Lovely Professional University, Phagwara, Punjab. India. \\ *Corresponding author's E-mail: sadak2020@gmail.com
}

Received: 11-02-2021; Revised: 15-04-2021; Accepted: 23-04-2021; Published on: 15-05-2021.

\section{ABSTRACT}

Nano materials such as nanoparticles, nano capsules, nano emulsions, nano suspensions, nano spheres, solid lipid nanoparticles, dendrimers, nano tubes, nano shells, nano wires, nano pores, and quantum dots have been used extensively for delivery of therapeutic agents. Protein nanocapsules have gained significance as vehicles for targeted drug delivery due to improved ease of administration, medical imaging, gene delivery, and increased half-life of drug. Nano materials and devices are used for addressing medical problems and have established enormous potential for, subcutaneous imaging without surgical incisions. They are used in imaging of liver, lymph nodes, bone marrow, monitoring of many serious illnesses, including cancer, CVS disorders, neurological disorders, HIV/AIDS, and diabetes. They are employed as solubilizing agent for water-insoluble drugs by emulsification and designing of essential oil protein complexes which paved the path for using nano materials in drug delivery with prolonged blood circulation times. PLGA and albumin nanoparticles are considered to be a potential drug carrier with good biodegradability, biocompatibility, and non toxic nature. Eucalyptus oil contains 1,8-Cineol and $\alpha$-pinene as chief ingredients.1 Eucalyptus oil is known for healing of inflammation, pruritus, abscesses, sepsis, bacterial infections, blisters, boils, chicken pox, congestion, fungal infections, herpes (cold sores) and ulcers. However, these essential oils are unstable, volatile, which limits their use for new formulations. Therefore, this study focuses on developing a PLGA- Albumin nano carrier for the encapsulation of essential oils. The effects of process parameters such as the effect of heat and the concentrations of polymers were investigated. Various physicochemical characterizations such as SEM, FTIR, EE, and UV spectroscopy, Invitro drug release studies were performed.2 Formulation F4 containing $0.6 \mathrm{mg} / \mathrm{mL}$ of PLGA and AL loaded eucalyptus loaded nanocapsules produced minimum-sized particles $(<300 \mathrm{~nm}$ ) with good encapsulation, improved stability, better efficiency and maximum invitro drug release.

Keywords: PLGA, Albumin, Essential oil, Eucalyptus oil, Nanocapsules.

QUICK RESPONSE CODE $\rightarrow$

DOI:

10.47583/ijpsrr.2021.v68i01.032

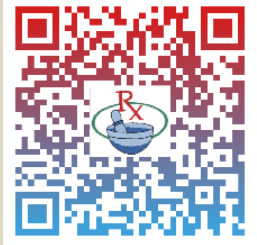

DOI link: http://dx.doi.org/10.47583/ijpsrr.2021.v68i01.032

\section{INTRODUCTION}

$\mathrm{N}$ anocapsules are vesicular systems made of a polymeric membrane which encapsulates an inner liquid core at the nanoscale with polymers. A nanocapsule consists of shell and a space in which preferred drug may be encapsulated. The size of nanocapsules varies from 5 to $1000 \mathrm{~nm}$, but in general they are $100-500 \mathrm{~nm} .{ }^{3}$ It is a submicroscopic colloidal drug transporter system. Nanocapsules can be prepared by using natural or synthetic polymers. In nanocapsules, drugs are placed into a core cavity, which provides safety from rapid degradation. It can be fabricated by diverse methods such as nanoprecipitation, emulsion diffusion, double emulsification polymer coating, and layer by layer method. ${ }^{4}$ Nanocapsule is composed of an aqueous or an oily core encircled by a thin polymer membrane. Nanocapsules are prepared from the aqueous solutions, by deposition of polymer layers onto a sacrificial template. For the assembly of the multilayer shell, interactions such as electrostatics, covalent bonding, hydrogen bonding, etc. can be used as the driving force. Dissolution of the sacrificial template leads to the formation of the hollow capsules. These hollow nanocapsules have the capacity to encapsulate a diverse type of materials such as drugs, catalysts, and biopolymers such as proteins and nucleic acids.

Applications of nano science and nanotechnology in health care led to the beginning of a novel field called nano medicine. Nanotechnology in drug delivery system provides an opportunity to deliver drugs for a pro-longed time with natural affinity. There has been an increased exploration of nanoparticles in the field of nanomedicine due to their exclusive physical and chemical properties. They are notorious to have high surface-free energy which makes them quite unstable. They attain stability by binding to biomolecules such as proteins that lessen the free surface energy. Polymeric nanocapsules can be made 
in specific sizes, shapes, and in reasonable quantities. They can be shaped as monodisperse particles with exactly distinct biochemical, electrical, optical, and magnetic properties. They can be customized to outfit the complexity of whatever application they are anticipated for, such as causing the release of the contents in response to a particular biomolecular triggering means in targeted drug delivery systems.

Nano particulate systems, dendrimers, micelles, liposomes, and nano emulsions are some of the nano carrier systems extensively utilized in delivery of drugs ${ }^{4}$. These nano carrier systems assist in improving bio distribution of drugs, solubility of hydrophobic substances, increasing the bioavailability, reducing the quantity of doses, enhancing drug targeting, and minimizing toxicity. The degradation and volatility of essential oils make them difficult to be used in pharmaceutical preparations. ${ }^{5}$ These disadvantages can be overcome to an extent by encapsulating these essential oils in nano carriers. There are various potential advantages of conjugating anticancer agents with nanoparticles, including targeted delivery and controlled release at diseased sites by changing their pharmacokinetic profile. Therefore, in present work, formulation of PLGA-AL nanocapsules was carried out with the goal of enhancing the physical stability of the essential oils by encapsulating them into the nanocapsules.

Albumin is an exceptional lyoprotectant for the solid form of nanomedicine and allows instant re-dispersion of dried out nanoparticles in injectable solutions. ${ }^{6}$ This advantage favors the increase of shelf-stable nanomedicine by shunning drug release and precipitate development. Albumin is circulated all over the body through blood flow, which has been utilized to resolve the total blood and plasma volume. In optimizing nanosystems, albumin is used for defined diagnosis and effective drug delivery in vivo. Albumin is a versatile protein carrier with several characteristics that make it an ultimate candidate for drug delivery, such as its availability, biocompatibility, biodegradability, lack of toxicity and immunogenicity. ${ }^{7}$ Therapeutic drugs have some disadvantages such as low solubility, low absorption and many side effects, which can be overcome using nanocarriers like albumin. Albumin is a constituent of human blood. It shows no immunogenicity as an endogenous protein (35-50 g/L in human serum) and ensures its biocompatibility for preparation of albuminbased nanoparticles. The half-life of albumin in human blood is about 19 days on average. Contrasting to free drugs, albumin presents a long half-life that helps improve the plasma area under curve (AUC) value, by this means maintain a blood drug concentration for a relatively long time. Albumin is distributed all over the body via blood flow, which has been utilized to determine the total blood and plasma volume.

Poly-lactic-co-glycolic acid (PLGA), an FDA approved copolymer, is one of the most exploited polymers in the design and formulation of drug delivery systems for biomedical applications owed to its biodegradability, bio safety, biocompatibility, versatility in formulation and functionalization. ${ }^{8}$ The structure of PLGA is given in fig.1. Supplementary, nanocarriers based on PLGA ensure an optimal bioavailability of the encapsulated drug by protecting it from premature degradation in the biological environment, providing a sustained and targeted delivery or facilitating intracellular penetration of bioactive compound and reducing the side effects. In this context, PLGA nanoparticles have been extensively used for various applications, such as: neuronal/cerebral disorders, cancer therapy, anti-inflammatory, cardiovascular or immune therapy, encapsulating a widely range of bioactive molecules (different drugs, proteins, vaccines, nucleic acids). Moreover, these nanosystems can be formulated for systemic (parenteral), oral and inhalable (pulmonary) administration offering the possibility to choose the best administration route, thus ensuring the optimal bioavailability of the system. PLGA-based nanoparticles have small size, high structural integrity, stability, tunable properties and versatility in surface functionalization.

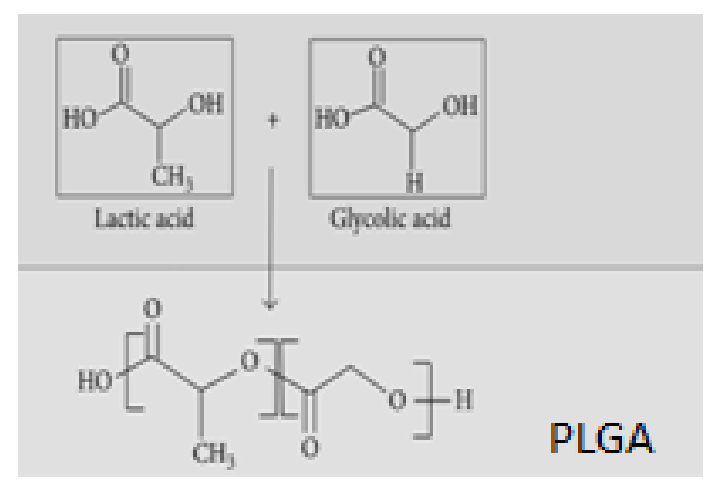

Figure 1: Structure of PLGA

Essential oils are volatile, aromatic liquids obtained from plants, herbs with curative and therapeutic activities. Essential oils are usually used in flavoring agents, perfumes, and pharmaceutical drugs. Of the many essential oils, eucalyptus oil is known for their high therapeutic potential. Eucalyptus oil is obtained by distillation of leaf of Eucalyptus, a genus of the plant belonging to family of Myrtaceae. ${ }^{9}$ The leaves of selected Eucalyptus species are steam distilled to extract eucalyptus oil. Eucalyptus oil has wide applications, as an antiseptic, repellent, flavoring agent, healing of inflammation, pruritus, abscesses, sepsis, bacterial infections, blisters, boils, chicken pox, congestion, fungal infections, herpes and ulcers.

\section{MATERIALS AND METHODS}

Albumin and PLGA were purchased from Sigma-Aldrich Company. Commercially available eucalyptus oil was purchased from SNN natural products. Thiazolyl blue tetrazolium bromide was purchased from Merck (Mumbai, Maharastra. India). Calcium chloride was purchased from JK pharmachem and other solvents used in this study were of analytical grade. The experiments were performed using deionized distilled water and were carried out in triplicates. 


\section{Preparation of essential oil-loaded PLGA-AL nanocapsules:}

Emulsification processes are employed in the preparation of nanocapsules in food, cosmetic, pharmaceutical and chemical industries. Essential oil loaded PLGA-AL nanocapsules were manufactured using simple protocol which consists of three steps: o/w emulsification, gelification and solvent removal. ${ }^{10}$ In brief, the o/w emulsion was made by dispersion of an ethanolic solution of eucalyptus oil into appropriate volumes of PLGA solution containing Tween 80 , with subsequent sonication. The emulsion was added drop wise to a suitable amount of calcium chloride and albumin solution, and the mixture was stirred for an additional $30 \mathrm{~min}$ and equilibrated overnight before removal of the solvent under reduced pressure.

During the development of nanocapsules, o/w nano emulsion was prepared by adding $20 \mathrm{~mL}$ aqueous PLGA solution of various concentrations. Formulations (F1 to F4) contain 0.3 to $0.6 \mathrm{mg} / \mathrm{mL}$ of PLGA with $1 \%(\mathrm{w} / \mathrm{v})$ Tween80. Subsequently, $0.6 \mathrm{~mL}$ of eucalyptus solution $(20 \mathrm{mg} /$ $\mathrm{mL}$ ) was added drop wise into this mixture. ${ }^{11}$ The $\mathrm{p}^{\mathrm{H}}$ of aqueous albumin solution was maintained at 5-5.5 to obtain nano-sized capsules. Mixtures were sonicated for 15 minutes. $5 \mathrm{~mL}$ of $0.7 \mathrm{mg} / \mathrm{mL}$ calcium chloride solution was added as stabilizing agent and the emulsion was stirred for 30 minutes. The emulsion was then combined with $4 \mathrm{~mL}$ of albumin solution $[0.6 \mathrm{mg} / \mathrm{mL}$ in $1 \%(\mathrm{v} / \mathrm{v})$ acetic acid] and stirred for the next 30 minutes. The $\mathrm{P}^{\mathrm{H}}$ of PLGA was maintained at 5 . The resulting suspension was equilibrated over night and solvent was removed to obtain nanocapsules. Various formulation of Eucalyptus oil loaded PLGA - Albumin nanocapsules is given in table 1.

Table 1: Formulations of Eucalyptus oil loaded PLGA Albumin nanocapsules

\begin{tabular}{|c|c|c|}
\hline Formulations & $\begin{array}{c}\text { Concentration of } \\
\text { albumin } \\
\text { (mg/mL) }\end{array}$ & $\begin{array}{c}\text { Concentration of } \\
\text { PLGA (mg/mL) }\end{array}$ \\
\hline F1 & 0.6 & 0.3 \\
\hline F2 & 0.6 & 0.4 \\
\hline F3 & 0.6 & 0.5 \\
\hline F4 & 0.6 & 0.6 \\
\hline
\end{tabular}

Optimization of process parameters:

To study the factors involved in the optimization of nanocapsules various factors were altered. Optimization of essential oil-loaded Al- PLGA nanocapsules was accomplished by varying the concentrations of PLGA $(0.3$ to $0.6 \mathrm{mg} / \mathrm{mL}$ ), by varying the effect of heating the albumin solution prior usage for removal of any impurity before use. ${ }^{12}$ Additionally, physicochemical characterization was performed to select the best formulation essential oil loaded nanocapsules.
Physicochemical characterization of nanocapsules: ${ }^{13}$

\section{Particle size and zeta potential:}

The particle size and zeta potential of nanocapsules were measured by Zetasizer Nano ZS (Malvern Instruments, UK). Zeta potential present information about the potential difference between the dispersion medium and the stationary layer of the fluid attached to the dispersed particle, which assist to know about the stability of the nano formulations. Measurements were made by means of aqueous diluted samples by using the principle of photon correlation spectrometry. This device also gives the measurement of particle-size distributions in the range between 200 and $500 \mathrm{~nm}$.

\section{Scanning electron microscopy:}

The surface morphology, shape of various nano materials can be scanned with a field emission scanning electron microscope (Philips XL-30 scanning electron microscope) at $15 \mathrm{kV}$. Approximately $5 \mathrm{~mL}$ of the nanocapsule sample was freeze dried and scanned using the scanning electron microscope.

\section{Fourier transform infrared spectroscopy:}

Oscillations of atoms in molecules were measured by Spectrum 3 FT-IR Spectrometer. These vibration spectroscopes present particulars regarding molecular vibrational energy levels. These energy levels are directly proportional to molecular structure, intermolecular interaction, and the chemical bonding. The Fourier transform infrared (FT-IR) spectra of nano formulations were examined using $\mathrm{KBr}$ pellet $[1 \%(\mathrm{w} / \mathrm{w})$ of product in $\mathrm{KBr}$ ] with a resolution of $4 \mathrm{~cm}^{-1}$ and 100 scans on Perkin Elmer spectrophotometer. It is a powerful tool for identifying diverse types of chemical bonds in a molecule by producing an infrared absorption spectrum. The FT-IR spectra of essential oil, bare PLGA-Al nanocapsules, and essential oil-loaded PLGA- nanocapsules were collected between 4000 and $800 \mathrm{~cm}^{-1}$.

\section{Encapsulation efficiency (EE) of nanocapsules: ${ }^{14}$}

Nanocapsules were alienated by centrifugation $(6000$ rpm) at $4^{\circ} \mathrm{C}$ for 10 minutes from the aqueous medium. The supernatant liquid was removed and $3 \mathrm{~mL}$ of methanol was added, vortexed well, and centrifuged for next 30 minutes. ${ }^{15}$ The supernatant liquid was collected and quantified spectrophotometrically. Using a standard curve that was plotted using different concentrations of albumin, the concentration of the eucalyptus oil was predicted. The encapsulation efficiency was calculated using the following equation: ${ }^{16}$

$$
\begin{gathered}
E E(\%)=(\text { Total amount of oil }- \text { Free oil }) / \text { Total amount of } \\
\text { oil } \times 100
\end{gathered}
$$

In vitro release studies: ${ }^{17}$

An invitro drug release study was carried out using the dialysis method. In this method, the dialysis bag was soaked in distilled water to wash the preservatives and 
rinsed with phosphate buffer saline (PBS) solution. The essential oil-loaded PLGA-AL nanocapsules were redispersed in $3 \mathrm{~mL}$ of PBS solution and loaded into the dialysis bag, enclosed by $50 \mathrm{~mL}$ of PBS containing $20 \%$ ethanol at $\mathrm{pH} 1.5$ and 7.4. The use of ethanol helps to decrease aggregation. ${ }^{18}$ The time-dependent drug release study at 0-12 hours was conducted. All sets of nanocapsules were incubated at $37^{\circ} \mathrm{C}$ under moderate agitation. At specific time intervals, $3 \mathrm{~mL}$ of the medium was collected and same amount of fresh medium was replaced. The samples were analyzed spectrophotometrically. The release was quantified as follows: Drug Release $(\%)=[$ Released oil/Total oil $] \times 100$

\section{Hemocompatibility assay:}

The hemocompatibility of essential oil loaded PLGA-Al nanocapsules can be carried out by hemolysis test. ${ }^{19}$ Blood was collected from a healthy human volunteer, with informed consent, into a tube containing anticoagulant solution of EDTA and incubated with $0.9 \%$ saline at temperature of $37^{\circ} \mathrm{C}$. Nanocapsules were added to diluted blood and incubated for 1 hour at $37^{\circ} \mathrm{C}$. Mixture of blood with distilled water was taken as the positive control and $0.9 \%$ saline with blood was taken as the negative control. After incubation the samples were centrifuged at 3000 rpm for 5 minutes to check hemocompaibiliy. ${ }^{20}$

\section{RESULTS AND DISCUSSION}

\section{Size and stability of nanocapsules:}

Essential oil-loaded PLGA-AL nanocapsules were prepared using the ionic-gelation procedure. Ethanol was used to dilute the essential oil. Particle size and stability were determined using the zetasizer. The following parameters were considered to check the stability of nanocapsules: a) heating of albumin solution b) without application of heat. Initially, the concentration of Albumin was kept constant and the concentration of PLGA was varied. For every concentration, the effect of heat was also observed and depicted Table 2 . In general, zeta potential values less than -30 $\mathrm{mV}$ and $+30 \mathrm{mV}$ for prepared nanocapsules are considered moderately stable. Preparations with zeta potential values less than $-40 \mathrm{mV}$ and $+40 \mathrm{mV}$ are considered stable formulation. Considering both particle size and stability, it was observed that the combination of $0.6 \mathrm{mg} / \mathrm{mL}$ and $0.6 \mathrm{mg} / \mathrm{mL}$ was stable preparation. Formulation F4 produced best results with particle sizes of $253.9 \mathrm{~nm}$ and zeta potential of $41.9 \mathrm{mV}$ without heating and $255.5 \mathrm{~nm}$ and zeta potential of $42.3 \mathrm{mV}$ with heating. The zeta potential was greater than $+35 \mathrm{mV}$ in oil loaded particles, indicating their good stability. However, consistent differences in particle size or zeta potential were not revealed due to the heating of solutions before the preparation process. A better particle size than previous reports was achieved mostly due to maintenance of $\mathrm{P}^{\mathrm{H}}$ at 5 . Within this $\mathrm{pH}$, a major contribution to the formation of complex and optimal interaction for the formation of smaller nanocapsules was observed. Therefore, the concentration of the polymers was optimized and the same concentration was used for further physicochemical characterization studies. Particle size and zeta potential of various formulations are tabulated in table 2 .

Table 2: Particle size and zeta potential of various formulations by application of heat and without application of heat

\begin{tabular}{|c|c|c|c|c|c|c|}
\hline \multirow[b]{2}{*}{ Formulation } & \multirow{2}{*}{$\begin{array}{l}\text { Concentration } \\
\text { of poly lactide } \\
(\mathrm{mg} / \mathrm{mL})\end{array}$} & \multirow{2}{*}{$\begin{array}{l}\text { Concentration } \\
\text { of albumin } \\
\text { (mg/mL) }\end{array}$} & \multicolumn{2}{|c|}{ By heating albumin } & \multicolumn{2}{|c|}{ Without heating albumin } \\
\hline & & & $\begin{array}{l}\text { Particle size } \\
\text { (nm) }\end{array}$ & $\begin{array}{c}\text { Particle } \\
\text { size }(\mathrm{nm})\end{array}$ & $\begin{array}{l}\text { Zeta potential } \\
\text { (mV) }\end{array}$ & $\begin{array}{c}\text { Zeta potential } \\
(\mathrm{mV})\end{array}$ \\
\hline $\mathrm{F} 1$ & 0.3 & 0.6 & 286.6 & 289.4 & 37.1 & 37.3 \\
\hline F2 & 0.4 & 0.6 & 279.5 & 288.9 & 38.9 & 38.0 \\
\hline F3 & 0.5 & 0.6 & 268.5 & 275.4 & 39.4 & 38.5 \\
\hline $\mathrm{F} 4$ & 0.6 & 0.6 & 255.5 & 253.9 & 41.9 & 42.3 \\
\hline
\end{tabular}

\section{Encapsulation Efficiency (EE):}

The EE\% was determined using ultraviolet visible spectroscopy. Using a standard graph, the concentrations of the eucalyptus oil were determined. Samples were analyzed in triplicates and it was found that Formulation F4 contains $96.9 \%$ of the eucalyptus oil. This suggests that carrier with $0.6 \mathrm{mg} / \mathrm{mL}$ of albumin and $0.6 \mathrm{mg} / \mathrm{mL}$ i.e., formulation F4 has more efficiency to encapsulate the essential oil. PLGA is very well suited for encapsulation of hydrophobic drugs. Encapsulation efficiency of various formulations is given in table 3 :
Table 3: Encapsulation efficiency of various formulations

\begin{tabular}{|c|c|}
\hline Formulation & Encapsulation Efficiency (\%) \\
\hline F1 & 78.3 \\
\hline F2 & 82.1 \\
\hline F3 & 91.2 \\
\hline F4 & 96.9 \\
\hline
\end{tabular}




\section{FT-IR analysis:}

To support the results of zeta potential and EE\% of nanocapsules, further studies were carried out by FT-IR analysis to know about the complex formation and interaction of major functional groups involved in the encapsulation of essential oil using PLGA-AL. These results helped us in confirming the presence of oil in prepared nanocapsules. This indicates the strong interaction between PLGA-AL and oil in nanocapsules. FTIR spectroscopy is widely used for studying the interactions between drug and excipients. An FTIR spectrum of albumin is shown in Fig. 2 which confirms the presence of characteristic groups. In the FTIR spectra of the albumin, the characteristic peaks were observed at $1650 \mathrm{~cm}-1$ ( $-\mathrm{C}=\mathrm{O}$ stretching) due to amide I band and 1530 $\mathrm{cm}-1$ ( $\mathrm{C}-\mathrm{N}$ stretch with $\mathrm{N}-\mathrm{H}$ bending mode) due to amide II band which shows the presence of protein. Because each of the secondary structure was associated with a characteristic hydrogen bonding pattern between amide $\mathrm{C}=\mathrm{O}$ and $\mathrm{N}-\mathrm{H}$ groups, it was expected that each type of secondary structure will give rise to characteristic amide I and II absorptions that allows determination of protein secondary structure by IR spectroscopy. Similarly the albumin nanocapsules show peak at $1510 \mathrm{~cm}-1$ which slightly shifts to the lower wavelength due to change in $\mathrm{N}$ $\mathrm{H}$ bond.

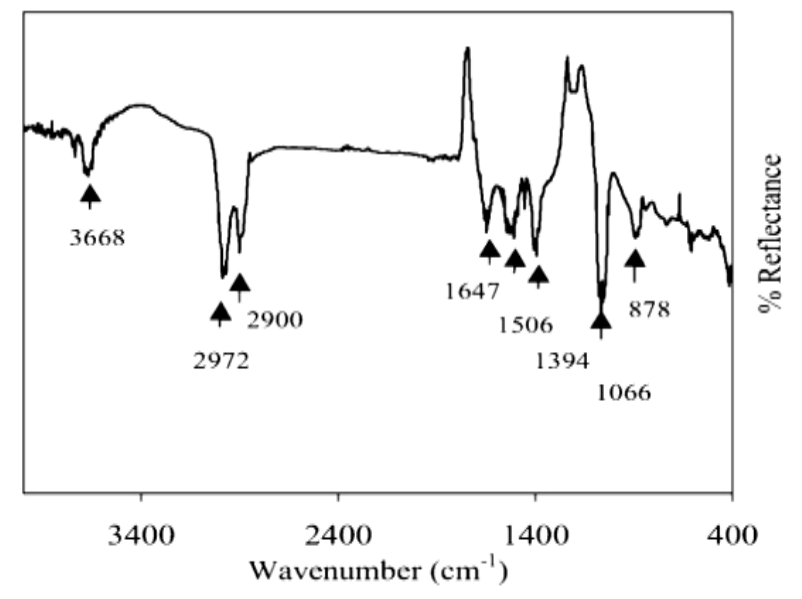

Figure 2: Fourier transform infrared spectra of albumin

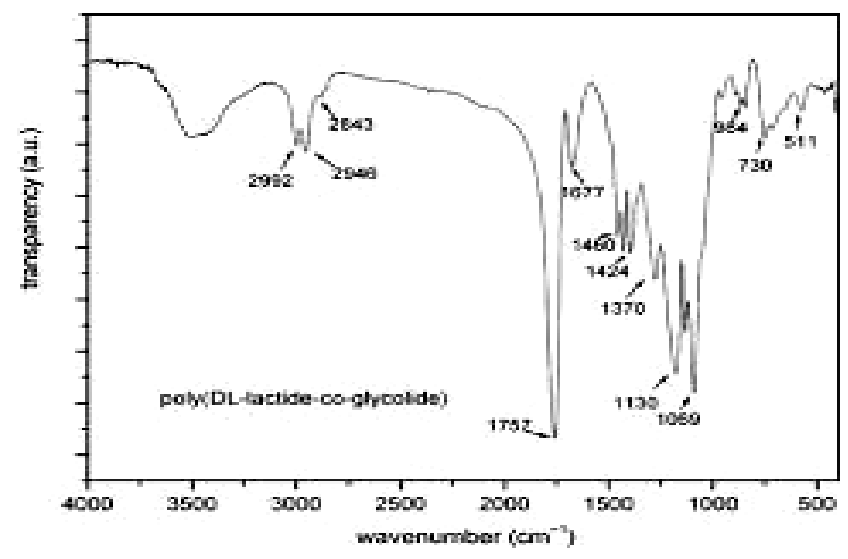

Figure 3: Fourier transform infrared spectra of PLGA
The characteristics peaks at 2843, 2948 and $2992 \mathrm{~cm}^{-1}$ were due to $\mathrm{C}-\mathrm{H}, \mathrm{C}-\mathrm{H}_{3}$ and $\mathrm{C}-\mathrm{H}_{2}$ functional group stretching vibration of PLGA are shown in fig.3.

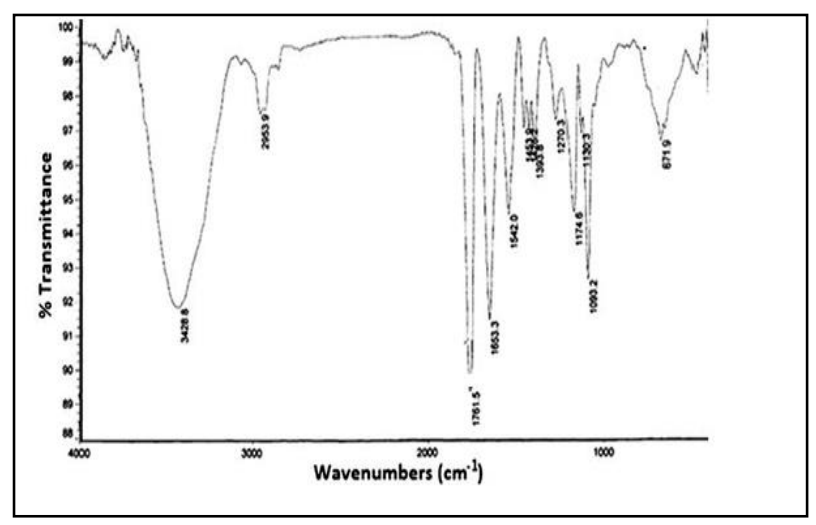

Figure 4: Fourier transform infrared spectra of PLGA -AL loaded nanocapsules

The characteristics peaks PLGA -AL loaded nanocapsules at indicates that there is no interaction between essential oil and polymers. It is also evident that the major peaks present in the spectra of bare nanocapsules are also prominent in the drug loaded nanocapsules. However, there are many minor peaks and some major peaks, which are formed due to the presence of essential oil as shown fig.4. The shift in the peaks indicates the possible interaction and successful encapsulation of oil into the PLGA-AL. Major peaks oil- loaded nanocapsules indicate the presence of $\mathrm{N}-\mathrm{H}, \mathrm{O}-\mathrm{H}, \mathrm{C}=\mathrm{O}$, and $\mathrm{CH}-\mathrm{OH}$ functional groups.

\section{Scanning electron microscopy:}

To obtain information about the morphology of the eucalyptus oil-loaded nanocapsules, scanning electron microscopy (SEM) analysis was performed. SEM analysis was carried out to visualize the size and shape of the nanocapsules. It can be inferred from fig. 5 that the essential oilloaded nanocapsules have spherical surface morphology and their mean size was found to be below $300 \mathrm{~nm}$.

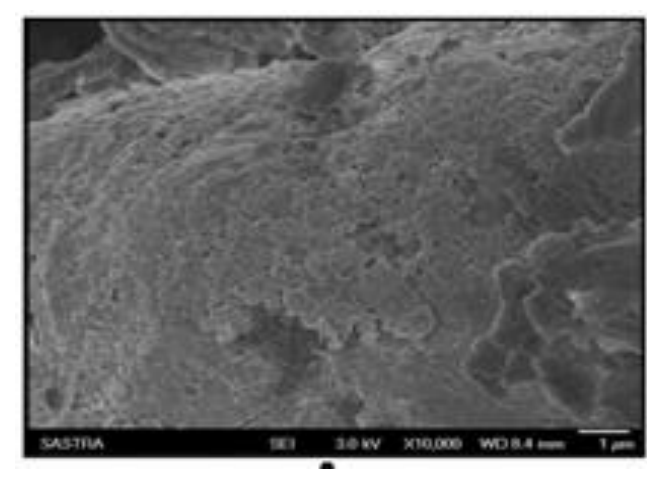

Figure 5: SEM image of Formulation F-4 nanocapsules

\section{In vitro drug release study:}

Release of essential oil-loaded PLGA-AL nanocapsules at $\mathrm{P}^{\mathrm{H}}$ 7.4 and 1.5 is depicted in fig.6. It was observed that at $\mathrm{pH}$ 7.4 , there was a better sustained release than at $\mathrm{P}^{\mathrm{H}}$ 1.5. At $\mathrm{pH} 7.4$, approximately $95 \%$ of the encapsulated eucalyptus oil was released for a period of 48 hours. At $\mathrm{pH} 1.5$, the drug 
release was less than $80 \%$ from oil loaded nanocapsules. This might be attributed to the formation of insoluble alginic skin at acidic $\mathrm{P}^{\mathrm{H}}$. This suggests that the drug release of PLGA- nanoparticles is $\mathrm{P}^{\mathrm{H}}$ sensitive and more oil will be released into the blood at $\mathrm{P}^{\mathrm{H}} 7.4$ than in the acidic stomach at $P^{H}$ 1.5. Therefore, more oil will be absorbed into the blood and in turn transported in the circulatory system to the site of infection more efficiently than when the oil is not encapsulated in the nanoparticles. Dissolution profile of various formulations of eucalyptus oil loaded nanocapsules is tabulated in table 4.

Table 4: Dissolution profile of various formulations of loaded nanocapsules

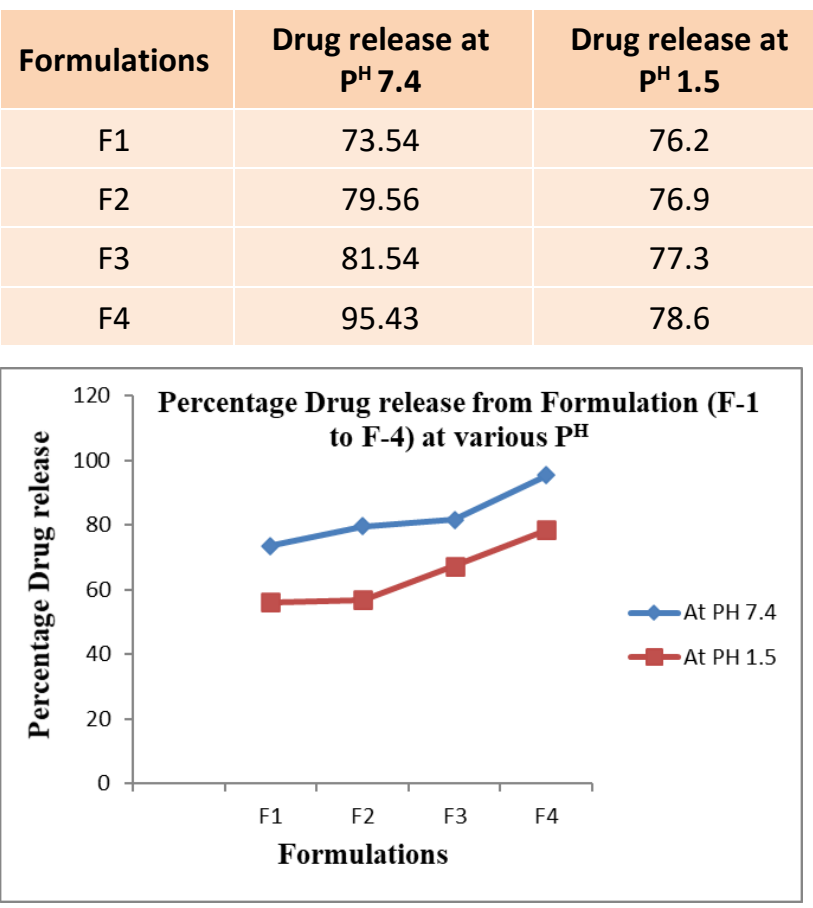

Figure 6: Percentage drug release from various formulation (F1 to F4) at various $\mathrm{P}^{\mathrm{H}}$

\section{Hemocompatibility test}

Essential oil-loaded nanocapsules were incubated with blood samples and the results were observed. The obtained results demonstrate that there was no lysis in the supernatant and the red blood cells settled down as pellets. Absence of lysis in the incubated sample demonstrated that this formulation was perfectly suitable for circulation in blood. Distilled water was used as the positive control to produce lysis of red blood cells, which contributes to the red supernatant. Saline, which was used as the negative control, did not show any hemolysis or toxicity to red blood cells, and therefore, the percentage of lysis was 0 . The blood samples incubated with oil-loaded nanocapsules showed results similar to those of saline, and therefore, it can be concluded that they have $0 \%$ hemolysis. Thus, it is proved that oil-loaded nanocapsules were hemocompatible and could be suitable for further drugdelivery applications. This may be due to the fact that encapsulated oil could reach to individual cells and deliver the oil to them. However, it was not possible for the free oil to reach the cells individually owing to its hydrophobicity. The nontoxic nature of carrier was confirmed with the viability of treated cells, which was $98 \%$.

\section{CONCLUSION}

PLGA- nanocapsules were synthesized using an ion gelation method and the size of the particles was below $290 \mathrm{~nm}$ shown good stability. Scanning electron microscopy (SEM) results suggest that the morphology of all the essential oilloaded nanocapsules was spherical. Encapsulation efficiency (EE) of Formulations (F1 to F4) was found to be $78.3 \%, \quad 82.1 \%, \quad 91.2 \%$ and $96.9 \%$ for oil-loaded nanocapsules. FT-IR results showed the functional groups played a major role in the interaction. The drug-release profile from formulation F-4 showed a slow and sustained release at neutral $\mathrm{P}^{\mathrm{H}}$ for 48 hours with $95 \%$ drug release. Hemolysis test was also confirmed from the clear supernatant in hemolysis assay indicates that prepared oilloaded PLGA-AL nanocapsules were hemocompatible, which suggests that they are a suitable carrier for further biomedical applications.

\section{REFERENCES}

1. Sebei K, Sakouhi F, Herchi W, Khouja ML, Boukhchina S. Chemical composition and antibacterial activities of seven Eucalyptus species essential oils leaves. Biol Res. 2015;48(1):7. doi:10.1186/0717-6287-48-7

2. Lin PC, Lin S, Wang PC, Sridhar R. Techniques for physicochemical characterization of nano materials. Bio technol Adv. 2014;32(4):711-726. doi:10.1016/j.biotechadv.2013.11.006

3. Kothamasu $\mathrm{P}$, Kanumur $\mathrm{H}$, Ravur $\mathrm{N}$, Maddu $\mathrm{C}$, Parasuramrajam R, Thangavel S. Nanocapsules: the weapons for novel drug delivery systems. Bioimpacts. 2012;2(2):71-81. doi:10.5681/bi.2012.011

4. Mora-Huertas CE, Fessi H, Elaissari A. Polymer-based nanocapsules for drug delivery. Int J Pharm. 2010 Jan 29;385(1-2):113-42. doi: 10.1016/j.ijpharm.2009.10.018.

5. Dhifi W, Bellili S, Jazi S, Bahloul N, Mnif W. Essential Oils' Chemical Characterization and Investigation of Some Biological Activities: A Critical Review. Medicines (Basel). 2016;3(4):25. doi:10.3390/medicines3040025

6. An FF, Zhang XH. Strategies for Preparing Albumin-based Nanoparticles for Multifunctional Bioimaging and Drug Delivery. Theranostics. 2017;7(15):3667-3689. doi:10.7150/thno.19365

7. Zhang $Y$, Sun $T$, Jiang $C$. Biomacromolecules as carriers in drug delivery and tissue engineering. Acta Pharm Sin B. 2018;8(1):34-50. doi:10.1016/j.apsb.2017.11.005

8. Makadia HK, Siegel SJ. Poly Lactic-co-Glycolic Acid (PLGA) as Biodegradable Controlled Drug Delivery Carrier. Polymers (Basel). 2011;3(3):1377-1397. doi:10.3390/polym3031377

9. Silva SM, Abe SY, Murakami FS, Frensch G, Marques FA, Nakashima T. Essential Oils from Different Plant Parts of Eucalyptus cinerea F. Muell. ex Benth. (Myrtaceae) as a Source of 1,8-Cineole and Their Bioactivities. Pharmaceuticals (Basel). 2011;4(12):1535-1550. doi:10.3390/ph4121535 
10. Makadia HK, Siegel SJ. Poly Lactic-co-Glycolic Acid (PLGA) as Biodegradable Controlled Drug Delivery Carrier. Polymers (Basel). 2011;3(3):1377-1397. doi:10.3390/polym3031377

11. Sobhani H, Tarighi P, Ostad SN, Shafaati A, Nafissi-Varcheh $\mathrm{N}$, Aboofazeli R. Formulation Development and Toxicity Assessment of Triacetin Mediated Nanoemulsions as Novel Delivery Systems for Rapamycin. Iran J Pharm Res. 2015;14(Suppl):3-21.

12. Ramalho MJ, Loureiro JA, Coelho MAN, Pereira MC. Factorial Design as a Tool for the Optimization of PLGA Nanoparticles for the Co-Delivery of Temozolomide and 06Benzylguanine. Pharmaceutics. 2019;11(8):401.doi:10.3390/pharmaceutics11080401

13. Ourique AF, Pohlmann AR, Guterres SS, Beck RC. Tretinoinloaded nanocapsules: Preparation, physicochemical characterization, and photostability study. Int J Pharm. 2008;352(1-2):1-4. doi:10.1016/j.ijpharm.2007.12.035

14. Yue PF, Lu XY, Zhang ZZ, et al. The study on the entrapment efficiency and in vitro release of puerarin submicron emulsion. AAPS PharmSciTech. 2009;10(2):376-383. doi:10.1208/s12249-009-9216-3

15. Livshits MA, Khomyakova E, Evtushenko EG, et al. Isolation of exosomes by differential centrifugation: Theoretical analysis of a commonly used protocol 2016;6:21447. doi:10.1038/srep17319

16. van Vuuren SF, du Toit LC, Parry A, Pillay V, Choonara YE. Encapsulation of essential oils within a polymeric liposomal formulation for enhancement of antimicrobial efficacy. Nat Prod Commun. 2010;5(9):1401-1408.

17. Natrajan D, Srinivasan S, Sundar K, Ravindran A. Formulation of essential oil-loaded chitosan-alginate nanocapsules. J Food Drug Anal. 2015;23(3):560-568. doi:10.1016/j.jfda.2015.01.001

18. Ofokansi K, Winter G, Fricker G, Coester C. Matrix-loaded biodegradable gelatin nanoparticles as new approach to improve drug loading and delivery. Eur J Pharm Biopharm. 2010;76(1):1-9. doi:10.1016/j.ejpb.2010.04.008

19. Lammari N, Louaer O, Meniai AH, Elaissari A. Encapsulation of Essential Oils via Nanoprecipitation Process: Overview, Progress, Challenges and Prospects. Pharmaceutics. 2020;12(5):431. doi:10.3390/pharmaceutics12050431

20. Bender EA, Adorne MD, Colomé LM, Abdalla DSP, Guterres SS, Pohlmann AR. Hemocompatibility of poly $(\varepsilon-$ caprolactone) lipid-core nanocapsules stabilized with polysorbate 80-lecithin and uncoated or coated with chitosan. Int J Pharm. 2012;426(1-2):271-279. doi:10.1016/j.jpharm.2012.01.051

Source of Support: None declared.

Conflict of Interest: None declared. 